\title{
The New Zeiss GeminiSEM 500 Meets the Needs of Challenging Biological Applications.
} Isabel Angert ${ }^{1}$, Christina Berger ${ }^{1}$, Martin Edelmann ${ }^{1}$, Robert Kirmse ${ }^{1}$, Kirk Czymmek $^{2}$, Alexander Thesen $^{1}$

1. Carl Zeiss Microscopy GmbH, Carl-Zeiss-Straße 22, 73447 Oberkochen, Germany

2. Carl Zeiss Microscopy, LLC, 1 Zeiss Drive, 10594 Thornwood, United States of America

Modern field emission scanning electron microscopy (FE-SEM) techniques are increasingly important for studying structural properties of cells and tissue. Typical applications include imaging of tissue by serial block face or array tomography methods (AT) [1,2]. In serial block face imaging a thin layer of the embedded sample is removed either mechanically or with a focused ion beam. Afterwards the fresh block face is imaged. In contrast for AT serial sections of the sample are produced in advance and mounted on a solid support (i.e. silicon wafer, glass). The individual sections are investigated in the FESEM afterwards. The imaging modes can vary from secondary electrons (SE) over back scattered electrons (BSE) detection to scanning transmission electron microscopy (STEM). Key performance parameters for biological imaging are optimum resolution and contrast, surface sensitivity, control of sample charging as well as the need of speeding up the data acquisition for high-throughput imaging.

The new ZEISS GeminiSEM500 meets these challenges with an improved low $\mathrm{kV}$ performance offering optimal resolution across the entire voltage range and a new variable pressure (VP) concept without significant loss in resolution when using in-lens detection. In addition GeminiSEM 500 features a broad detector portfolio ranging from efficient parallel in-lens SE and energy selective backscattered (EsB) detection over backscatter detection on a five sector diode detector to STEM detection on a diode based detector with real annular design (aSTEM).

Here we demonstrate a variety of application examples that highlight the benefits of the new system. The new low $\mathrm{kV}$ lens in the GeminiSEM 500 enables optimum resolution without biasing the stage (e.g 1.1 nm @1 kV, 1.2 nm @ 500V). Lower electron energies are particularly important for blockface imaging or nano-tomography to avoid beam damage of deeper layers that will be exposed next. Using the new Zeiss backscattered detector even at $1.5 \mathrm{kV}$ enables the detailed structural investigation of biological tissue with optimal contrast (Fig. 1A). Even at $50 \mathrm{~V}$ surface structures can be resolved in great details as shown at the example of an uncoated moth wing (Fig. 1B). Using such extremely low energies even the most challenging non-conductive samples can be investigated without charge accumulation. At higher beam energies needed to resolve structures below the surface the new NanoVP mode from Zeiss comes into play. The pressure in the specimen chamber can be adjusted from 1 to about $150 \mathrm{~Pa}$ and a differential pumping aperture is inserted below the objective lens which reduces the scattering of the electrons in the gas allowing in-lens detection (Fig. 1C) without considerable loss of resolution and increasing VPSE detection efficiency.

Transmission imaging mode in the scanning electron microscope (STEM) is a widely applied method for imaging transmissible samples often resulting in higher resolution than classical SE or BSE imaging mode [3]. The ZEISS aSTEM detector provides excellent contrast and dynamic range (Fig. 1D) and due to its design with concentric diode layout it allows bright-field (BF), annular dark-field (DF) and highangle annular dark-field (HAADF) detection. The detail that can be obtained in STEM mode is close to the limit in the amount of detail that can be expected from conventionally prepared, chemically fixed and plastic embedded samples. 


\section{References:}

[1] P Schneider et al. Bone 49 (2011) p. 304-311.

[2] M Kuwajima et al., PLoS One 8(3): e59573. (2013)

[3] KD Micheva and SJ Smith, Neuron 55 (2007) p. 25-36.
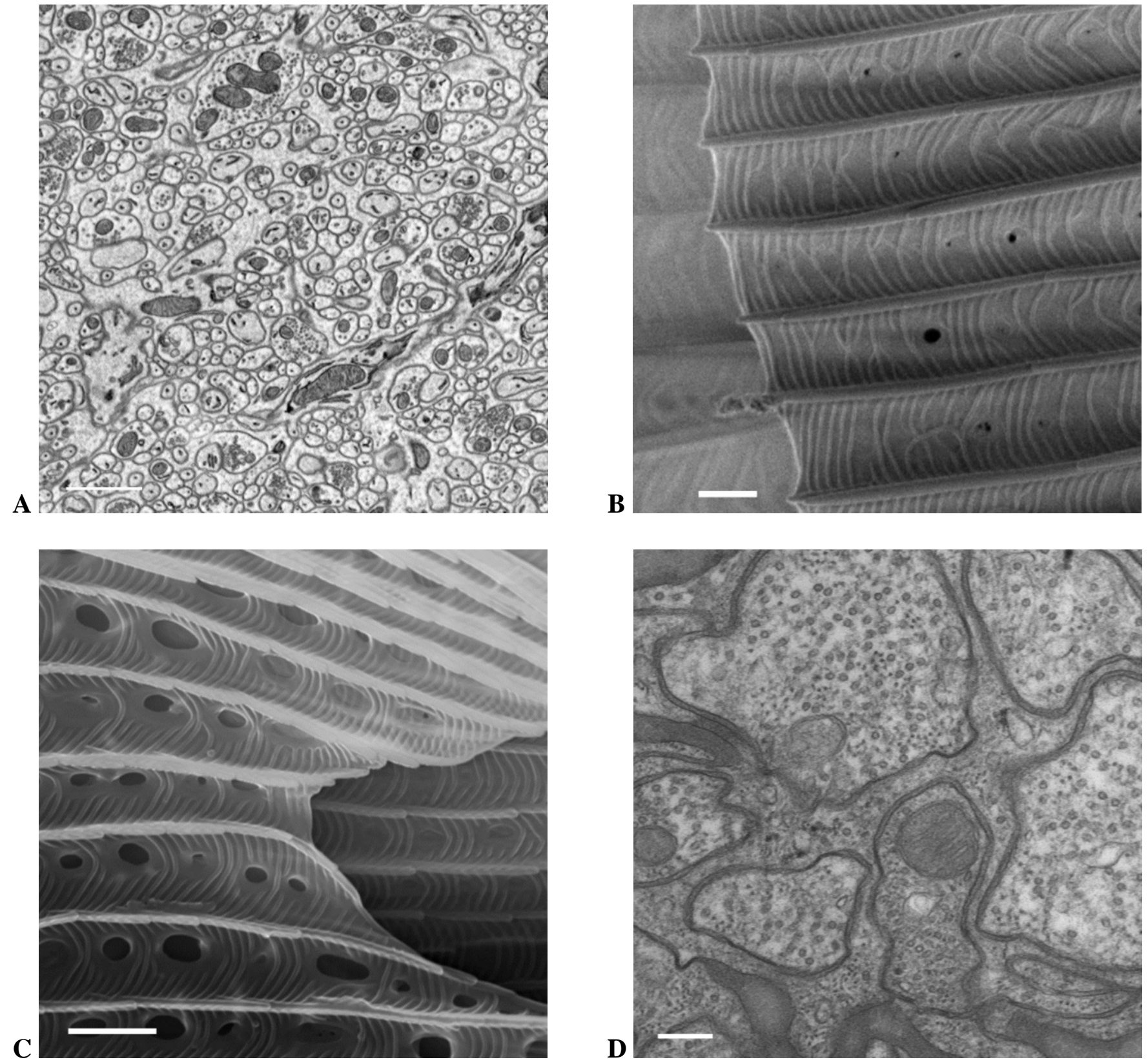

Figure 1. A: Image of brain tissue with a backscattered detector at $1.5 \mathrm{kV}$ primary energy with $10 \mathrm{~nm}$ pixel size, scale $1 \mu \mathrm{m}$. B: Uncoated moth wing imaged at $50 \mathrm{eV}$ with in-lens detection. This shows highest surface sensitivity and imaging of non-conductive samples, scale $1 \mu \mathrm{m}$. C: Uncoated moth wing imaged at $20 \mathrm{keV}$ with in-lens detection. With the nanoVP mode the pressure at the sample was set to 50 Pa, scale $1 \mu \mathrm{m}$. D: STEM image of brain tissue at $28 \mathrm{kV}$ in BF mode, scale $200 \mathrm{~nm}$. 